\title{
USO DE DIAMINO FLUORETO DE PRATA EM CRIANÇAS ATENDIDAS EM UMA UNIDADE BÁSICA dE SAÚdE dO NORTE dO PARANÁ
}

\author{
Use of diamino silver fluoride in children of a Basic \\ Health Unit located in north of the state of Paraná
}

Caroline Delmaschi Ramos ${ }^{1}$, Carolina Veloso Lima², Mayara dos Santos Noronha ${ }^{3}$, Daylana da Silva Pacheco ${ }^{4}$, Bárbara Emanoele Costa Oliveira ${ }^{5}$, Pablo Guilherme Caldarelli ${ }^{6}$

\begin{abstract}
1. Universidade Estadual de Londrina - UEL. ORCID: http://orcid.org/0000-0001-8158-3137
2. Universidade Estadual de Campinas - UNICAMP. ORCID: http://orcid.org/0000-0002-7575-3651

3. Universidade Estadual de Campinas - UNICAMP. ORCID: http://orcid.org/0000-0003-3963-7503

4. Universidade Estadual de Campinas - UNICAMP. ORCID: http://orcid.org/0000-0002-1690-6451

5. Universidade Estadual de Campinas - UNICAMP. ORCID: http://orcid.org/0000-0002-6693-360X

6. Universidade Estadual de Londrina - UEL. ORCID: http://orcid.org/0000-0002-4589-9713
\end{abstract}

CONTATO: Pablo Guilherme Caldarelli | Rua Juiz de Fora, 230 | Jardim Hedy | Londrina | Paraná | Brasil | CEP 86062-680 | E-mail: pablocaldarelli@otmail.com

COMO CITAR Ramos CD, Lima CV, Noronha MS, Pacheco DS, Oliveira BEC, Caldarelli PG . Uso de diamino fluoreto de prata em crianças atendidas em uma unidade básica de saúde do Norte do Paraná. R. Saúde Públ. 2018 Jul., 1(1):xx-xx.

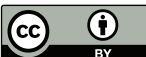

COPYRIGHT Esta obra é disponibilizada nos termos da Licença Creative Commons - 4. 0 Internacional. É permitida a reprodução parcial ou total desta obra, desde que citada a fonte.

RESUMO O presente estudo avaliou o uso e a aceitação de diamino fluoreto de prata (DFP) em crianças atendidas em uma Unidade Básica de Saúde (UBS) do Norte do Paraná, bem como o cumprimento do protocolo municipal para aplicação do DFP. Prontuários $(n=43)$ de crianças de 0 a 5 anos residentes na área de abrangência da UBS foram analisados. Foram coletadas informações sobre idade da criança, utilização e indicação do DFP, dentes submetidos ao tratamento e quantidade de reaplicações. Observou-se que o DFP foi aplicado em $79 \%$ das 
crianças devido à Cárie Precoce na Infância (CPI), 60\% dos dentes que receberam a aplicação do DFP eram posteriores e $86 \%$ dos responsáveis aceitaram utilizar o DFP. Portanto, conclui-se que o DFP tem sido utilizado no serviço público do Paraná e que o procedimento é aceito pela maioria dos responsáveis das crianças, mas o cumprimento do protocolo municipal para aplicação do DFP ainda merece atenção.

PALAVRAS-CHAVE: Cárie Dentária. Flúor. Prevenção e Controle.

\begin{abstract}
This study evaluated the use and acceptance of silver diamine fluoride (SDF) in children treated in a Basic Health Unit (UBS) in the North of the state of Paraná, as well as the compliance with the municipal protocol for SDF application. Medical records $(n=43)$ of children aged from 0 to 5 years old residing in the area covered by the UBS were analyzed. Information about child's age, use and indication of SDF, teeth treated, and number of SDF reaplications were collected. It was observed that SDF was used in $79 \%$ of children due to Early Childhood Caries (ECC), 60\% of the teeth treated were posterior teeth, and $86 \%$ of the children's tutors accepted SDF use. Therefore, SDF has been used in Paraná's public health service, the treatment was well accepted by most children's tutors, but the compliance with the municipal protocol for SDF treatment still needs attention.
\end{abstract}

KEYWORDS: Dental Caries. Fluorine. Prevention and Control.

\section{INTRODUÇÃO}

$\mathbf{0}$

diamino fluoreto de prata (DFP) é um produto de ação tópica usado em vários países como uma alternativa para o tratamento da cárie dentária ${ }^{12}$. Trata-se de uma solução alcalina composta por um complexo entre amônia, prata e fluoreto $\left(\mathrm{AgF}\left(\mathrm{NH}_{3}\right)_{2}\right)$. Assim, o ín fluoreto atua interferindo com o processo fisico-químico do desenvolvimento da lesão de cárie, enquanto a prata tem efeito bactericida ${ }^{3} \mathrm{e}$ atua como um antimicrobiano ${ }^{2.4}$. Adicionalmente, sugere-se que o DFP poderia ser capaz de proteger a matriz colágena da dentina contra a degradação, aumentando sua capacidade de remineralização 2.5 . Desta forma, a lesão tratada poderia aumentar a densidade mineral e a dureza à medida que a profundidade da lesão diminui ${ }^{3}$.
Nesse contexto, o DFP tem sido utilizado de maneira eficaz na prática clínica ${ }^{6.78,9}$. Considerando que o uso deste produto é considerado nãoinvasivo, indolor e de fácil aplicação, ele tem sido recomendado para crianças na primeira infância, com alta atividade cárie e não cooperativas ${ }^{10.11}$. No estado do Paraná, historicamente sua utilização encontra-se relacionada ao surgimento e estruturação do Programa de Atenção Precoce à Saúde Bucal (Bebê-Clínica), ocorrido em 1985 por meio do curso de Odontologia da Universidade Estadual de Londrina (UEL) e da Financiadora de Estudos e Projetos (FINEP) ${ }^{6}$.

Embora o DFP seja efetivo no controle de lesões de cárie, sua aplicação causa um manchamento superficial do esmalte, exibindo 
uma pigmentação escura ${ }^{12,13}$. Portanto, o critério estético pode ser considerado um fator limitante na escolha e utilização desse material. Apesar de diversos estudos abordarem a utililização do DFP 1,2.5.9, é necessário entender de que forma os pais e/ou responsáveis lidam com esse tipo de tratamento, tendo em vista que sua aplicação pode ocasionar problemas de caráter social devido ao comprometimento estético ${ }^{12,13}$.

O município de Londrina-PR apresenta um Protocolo Municipal de Saúde Bucal ${ }^{14}$ o qual contempla a utilização do DFP em dentes posteriores. De acordo com este manual, o uso do DFP é indicado para tratamento de crianças com alto risco de cárie, biofilme dental visível e em situações onde a escovação noturna e higiene bucal após amamentação são negligenciados. Considerando as características e mecanismo de ação do DFP bem como o protocolo estabelecido, observou-se a necessidade de conhecer como esse produto tem sido utilizado na prática clínica dos serviços públicos de saúde bucal do município.

Dessa forma, o objetivo deste estudo foi avaliar o uso e a aceitação de diamino fluoreto de prata (DFP) em crianças atendidas em uma Unidade Básica de Saúde (UBS) do Norte do Paraná, bem como o cumprimento do protocolo municipal para aplicação do DFP.

\section{METODOLOGIA}

O presente estudo foi aprovado pelo Comitê de Ética em Pesquisa envolvendo Seres Humanos da Universidade Estadual de Londrina (CEP/UEL). parecer n. 1.154.455/2015, atendendo aos requisitos estabelecidos pela Resolução $N^{\circ} 466.503$ do Conselho Nacional de Saúde / Ministério da Saúde (CNS/MS).

Foi realizada uma pesquisa quantitativa, restrospectiva e transversal. Para isso, dentre as duas Unidades Básicas de Saúde que encontramse vinculada à Residência Multiprofissional em Saúde da Família (RMSF) da Universidade Estadual de Londrina (UEL) realizou-se um sorteio. Dessa forma, a pesquisa foi realizada no Centro de Saúde Dr. Walter Zamarian, localizado no Parque das Indústrias, região Sul do município de LondrinaPR (UBS PIND). Essa Unidade Básica de Saúde conta com uma equipe completa de saúde bucal do município, para atendimento infanto-juvenil e gestantes, e dois cirurgiões-dentistas da RMSF para atendimento na lógica da Estratégia Saúde da Família (ESF).

Neste cenário, foram analisados 2.523 prontuários. A análise foi realizada por apenas um pesquisador, previamente calibrado. Os prontuários correspondiam a crianças atendidas na UBS PIND entre os meses de janeiro de 2012 a abril de 2016. Somente os dados dos prontuários contendo registro de indicação e/ou utilização do DFP em crianças entre 0 e 5 anos de idade foram coletados $(n=43)$.

Além da indicação/utilização do DFP, foram obtidos dados sobre idade da criança, motivo para a indicação do DFP pelo profissisonal, elementos dentários em que o DFP foi aplicado e a utilização do termo de autorização para aplicação do DFP. Além disso, em casos de não autorização, foi verificado se no prontuário encontrava-se descrito o motivo pelo qual essa decisão foi tomada. Os dados coletados foram tabulados pelo software Microsoft Excel ${ }^{\circledR}$ e tratados por estatística descritiva, com números absolutos e porcentagem.

\section{RESULTADOS}

Foi observado que a maioria (88,4\%) dos pais e/ou responsáveis aceitou o tratamento com a aplicação do DFP. A indicação de aplicação do DFP em aproximadamente $80 \%$ das crianças foi devido à presença da cárie precoce de infância (CPI) (Tabela 1).

Nenhuma criança de 0 a 1 ano teve indicação para o uso do DFP. Por outro lado, o DFP foi aplicado com maior frequência em crianças com idade entre 3 e 4 anos (Tabela 2).

A reaplicação do DFP foi realizada em $62,9 \%$ das crianças. A frequência observada foi 
Tabela 1. Indicação da aplicação do DFP em dentes de crianças atendidas na UBS PIND, Londrina-PR.

\begin{tabular}{l|c}
\hline \multicolumn{1}{c|}{ INDICAÇÃo } & $\begin{array}{l}\text { QUANTIDADE DE } \\
\text { CRIANÇAS N (\%) }\end{array}$ \\
\hline $\begin{array}{l}\text { Cárie precoce de } \\
\text { infância }\end{array}$ & $34(79,1 \%)$ \\
$\begin{array}{l}\text { Não aceitação de outro } \\
\text { tipo de tratamento }\end{array}$ & $8(18,6 \%)$ \\
$\begin{array}{l}\text { Alto risco à cárie (dieta } \\
\text { incorreta e má higiene } \\
\text { bucal) }\end{array}$ & $1(2,3 \%)$ \\
\hline
\end{tabular}

Fonte: Elaborado pelos autores (2018)

Tabela 2. Aplicação de DFP em crianças atendidas na UBS em Londrina-PR, de acordo com a idade e grupo de dentes.

\begin{tabular}{l|c}
\hline APLICAÇÃO DO DFP & $\begin{array}{c}\text { QUANTIDADE DE } \\
\text { CRIANÇAS N (\%) }\end{array}$ \\
\hline Idade (anos) & $2(4,7 \%)$ \\
2 anos & $17(39,5)$ \\
3 anos & $18(41,9 \%)$ \\
4 anos & $6(13,9 \%)$ \\
5 anos & $4(9,3 \%)$ \\
\hline Grupo de dentes & $26(60,5 \%)$ \\
Anteriores & $3(7 \%)$ \\
Posteriores & $10(23,2 \%)$ \\
$\begin{array}{l}\text { Anteriores e } \\
\text { posteriores } \\
\text { Sem informação no } \\
\text { prontuário }\end{array}$ & \\
\hline
\end{tabular}

Fonte: Elaborado pelos autores (2018)

de $2,3 \%$ para uma reaplicação, 32,6\% para duas reaplicações, $7 \%$ para três reaplicações e 21\% para quatro reaplicações.

A maioria dos cirurgiões-dentistas (86\%) utilizou um termo de autorização no qual os pais e/ ou responsáveis permitiram a aplicação do DFP nos dentes das crianças. No entanto, ressalta-se que uma parcela dos profissionais realizou a aplicação do DFP mesmo sem assinatura do termo (14\%).

\section{DISCUSSÃO}

Os resultados deste estudo mostram a aceitação dos pais/responsáveis em relação à aplicação do DFP nos dentes decíduos. Embora o produto apresente limitações estéticas ${ }^{12,13}$, o DFP age no controle da lesão de cárie, e consequentemente, preservação do tecido denta|2,3,15,16. Trata-se de uma opção viável para o controle de lesões de cárie cavitadas na dentição decídua ${ }^{16}$, por se tratar de um produto com aplicação simples, rápida e de baixo custo ${ }^{12}$, sendo efetivo em evitar a progressão dessas lesões ${ }^{1,2,16}$.

Conforme observado, a principal indicação para a aplicação do DFP foi a presença da cárie precoce na infância (CPI). Esta condição clínica afeta crianças menores de 3 anos e é sinalizada por qualquer superfície amolecida em sua dentição ${ }^{17}$ Por se tratar da doença cárie, seu desenvolvimento depende da formação de biofilme na superfície dental frequentemente exposta à carboidratos da dieta ${ }^{18}$, principalmente a sacarose. Aliado a isso, a falta de higiene bucal, o aumento da idade, além de fatores socioeconômicos e culturais nos quais a criança está inserida, podem modular o desenvolvimento da doença $a^{19,20}$.

Ressalta-se que a maior parte das aplicações de DFP foi feita em crianças com idade de 3 e 4 anos, possivelmente pelo fato de já apresentarem a dentição decídua completa e já possuirem uma dieta cariogênica. Além disso, a aplicação ocorreu com maior frequência nos dentes posteriores (60,5\%). Devido a aplicação ser realizada em dentes posteriores, essa aceitação do tratamento pode ser aumentada, independente da pigmentação que o DFP causa ao dente ${ }^{13}$. Esse comportamento devese ao fato dos pais relatarem que os benefícios do uso do diamino sobrepõem-se à sua capacidade de pigmentação ${ }^{12,13}$. Além disso, observou-se que além dos dentes posteriores, DFP tem sido aplicado em dentes anteriores, em divergência com o preconizado pelo protocolo do município de Londrina-PR ${ }^{14}$, que indica a aplicação de verniz fluoretado. Entretanto, mesmo quando aplicado nos dentes anteriores, os pais das crianças demonstram preferir o DFP 
ao invés de procedimentos mais invasivos que necessitam de anestesia geral ou sedação, como os casos de crianças não cooperativas ${ }^{13}$.

De acordo com protocolo utilizado no município de Londrina-PR ${ }^{14}$, o tratamento com DFP deve ser realizado com quatro aplicações em sessões consecutivas, com intervalo de uma semana entre elas, também conhecido como "fluorterapia intensiva" ou "tratamento de choque". No entanto, os resultados encontrados mostram que uma baixa porcentagem de dentistas (21\%) realizou as quatro aplicações preconizadas no protocolo. Nesse contexto, torna-se importante destacar que esse tipo de recomendação se mostra eficiente principalmente devido aos retornos semanais do paciente, servindo como um fator motivacional na manutenção do cuidado por pais e responsáveis. Porém, deve-se ressaltar que não existe um protocolo universal para aplicação tópica de fluoretos. Assim, cabe ao cirurgião-dentista utilizar os produtos de aplicação profissional de flúor da forma que melhor atender as necessidades de seu paciente ou as possibilidades de utilização no contexto em que está inserido²1.

Ademais, foi possivel observar a ausência de informações no preenchimento dos prontuários odontológicos, quanto à descrição dos procedimentos diários. Segundo o Conselho Federal de Odontologia $(C F O)^{22}$, a evolução do tratamento deve acontecer com anotações de todos os procedimentos realizados, relato conciso dos dentes e faces coronárias ou regiões envolvidas, materiais utilizados e data de realização. Assim, para que o procedimento possa ser realizado, o cirurgião-dentista deve solicitar ao responsável pela criança, a assinatura do termo de autorização. Caso o responsável não concorde com a aplicação do DFP, um termo de não autorização deve ser assinado. Os resultados encontrados nesse estudo mostraram que a maior parte dos profissionais (86\%) utilizou o termo de autorização, mas 14\% dos cirurgiões-dentistas realizaram a aplicação do DFP mesmo sem assinatura do termo.

Destaca-se que o uso e indicação do DFP devem ser esclarecidos pelo cirurgião-dentista aos pais/responsáveis, abordando seus benefícios, forma de aplicação e efetividade para o controle das lesões de cárie. Além disso, os responsáveis devem estar cientes da limitação estética oferecida pelo produto. Assim, para segurança do profissional, é imprescindivel a ultilização e assinatura do termo de autorização ou não autorização, como instrumento de defesa legal.

\section{CONCLUSÃO}

Conclui-se que o diamino fluoreto de prata é utilizado pelos dentistas da Unidade Básica de Saúde e tem sido bem aceito pelos pais e responsáveis das crianças. Contudo, o cumprimento do protocolo municipal para aplicação do DFP ainda merece atenção, assim como a ultilização de termo de autorização como um instrumento de defesa legal e a evolução dos prontuários.

\section{REFERÊNCIAS}

1. Contreras V. Toro MJ, Elías-Boneta AR, Encarnación-Burgos A. Effectiveness of silver diamine fluoride in caries prevention and arrest: a systematic literature review. Gen Dent. 2017;65(3):22-9.

2. Zhao IS, Gao SS, Hiraishi N, Burrow MF, Duangthip D, Mei ML, Lo EC, Chu $\mathrm{CH}$. Mechanisms of silver diamine fluoride on arresting caries: a literature review. Int Dent J. 2018 Apr;68(2):67-76.

3. Mei ML, Ito L, Cao Y, Li QL, Lo ECM, Chu CH. Inhibitory effect of silver diamine fluoride on dentine demineralisation and collagen degradation. Journal of Dentistry. 2013:41(9):809-17.

4. Wu MY, Suryanarayanan K, van Ooij WJ, Oerther DB. Using microbial genomics to evaluate the effectiveness of silver to prevent biofilm formation. Water Sci Technol. 2007:55(8-9):413-9.

5. Mei ML, Chu CH, Low KH, Che CM, Lo EC. Caries arresting effect of silver diamine fluoride on dentine carious lesion with $\mathrm{S}$. mutans and L. acidophilus dual-species cariogenic biofilm. Medicina Oral, Patología Oral y Cirugía Bucal. 2013;18(6):e824-e831.

6. Baldani MHP, Lopes CMDL, Kriger L, Matsuo T. A Odontologia para bebês no Estado do Paraná, Brasil - Perfil do programa de atenção precoce à saúde bucal. J Bras Odontopediatr Odontol Bebê. 2003:6(31):210-16.

7. Mei ML, Lo EC, Chu CH. Clinical Use of Silver Diamine Fluoride in Dental Treatment. Compend Contin Educ Dent. 2016 Feb;37(2):938; quiz100.

8. Chibinski AC, Wambier LM, Feltrin J, Loguercio AD, Wambier DS, Reis A. Silver Diamine Fluoride Has Efficacy in Controlling Caries Progression in Primary Teeth: A Systematic Review and MetaAnalysis. Caries Res. 2017:51(5):527-541 
9. Crystal YO, Marghalani AA, Ureles SD. Wright JT, Sulyanto R, Divaris K, Fontana M, Graham L. Use of Silver Diamine Fluoride for Dental Caries Management in Children and Adolescents, Including Those with Special Health Care Needs. Pediatr Dent. 2017 Sep 15:39(5):135-145.

10. Chu CH, Lo EC. Promoting caries arrest in children with silver diamine fluoride: a review. Oral Health Prev Dent. 2008;6(4):31521.

11. Rosenblatt A, Stamford TCM, Niederman R. Silver diamine fluoride: a caries "silver-fluoride bullet". Journal of Dental Research. 2009:88(2):116-25.

12. Sousa FSO, Santos APP, Barja-Fidalgo F, Oliveira BH. Evidence-based pediatric dental practice within the clinician's reach: the case of the esthetic effect of topical silver diamine fluoride for caries control in primary dentition. Rev Gaúcha de Odontol. 2016:64(4):369-75

13. Crystal YO, Janal MN, Hamilton DS, Niederman R. Parental perceptions and acceptance of silver diamine fluoride staining. J Am Dent Assoc. 2017 Jul;148(7):510-518.e4.

14. Londrina. Prefeitura Municipal. Manual de Saúde Bucal. 1 ed. Secretaria Municipal de Saúde. Paraná: Londrina; 2009.

15. Chu CH, Lo ECM, LO, Lin HC. Effectiveness of silver diamine fluoride and sodium fluoride varnish in arresting dentin caries in Chinese pre-school children. J Dent Ress. 2002;81:767-70.

16. Chu $\mathrm{CH}$, Lo EC. Promoting caries arrest in children with silver diamine fluoride: a review. Oral Health Prev Dent. 2008:6(4):31521.

17. AAPD. Definition of early childhood caries (ECC). Am Acad Pediatr Dent [Internet]. 2008;4(age 3):1. Available from: http:// www.aapd.org/assets/1/7/D_ECC.pd

18. Fejerskov O. Changing paradigms in concepts on dental caries: consequences for oral health care. Caries Res. 2004 MayJun;38(3):182-91.

19. Costa EL, Ferreira PVC, Oliveira BEC, Portugal RP, Rodrigues VP. Costa JF. Socioeconomic, Nutritional and Behavioral Factors Associated with Severe Childhood Caries in Children Aged 1836 Months. Pesquisa Brasileira em Odontopediatria e Clínica Integrada (Impresso). , v.14, p.79-87, 2014.

20. Anil S, Anand PS. Early Childhood Caries: Prevalence, Risk Factors, and Prevention. Front Pediatr. 2017 Jul 18:5:157. doi: 10.3389/fped.2017.00157. eCollection 2017

21. Cury JA, Tenuta LMA. Uso de fluoreto em Odontologia restauradora fundamentado em evidências. In: Baratieri LN, editores. Odontologia Restauradora - Fundamentos e Possibilidades. 2.ed. Grupo Gen: São Paulo; 2015.

22. Brasil. Resolução CFO n 118, de 11 de maio de 2012. Revoga o Código de Ética Odontológica aprovado pela Resolução CFO42/2003 e aprova outro em substituição. Diário Oficial da União, Brasília, 2012; 14 jun., seção 1, nº 114, p.118. 